\title{
ENERGY EFFICIENT ROUTING IN WIRELESS SENSOR NETWORKS BASED ON QOS
}

\author{
Syed Mohd Ali \\ Research Scholar ECE dept., JNTUH, Kukatpally, Hyderabad, India \\ Dr Syeda Gauhar Fatima \\ Professor ECE dept, Deccan College of Engineering and Technology \\ Darussalam, Hyderabad, India \\ Syeda Kausar Fatima \\ Associate Professor, Shadan College of Engineering and Technology, Hyderabad, India

\section{Dr Syed Abdul Sattar} \\ Principal, Nawab Shah Alam College of Engineering and Technology, Hyderabad, India
}

\begin{abstract}
ABTRACT
Energy is a serious resource parameter in Wireless Sensor Networks. Utilizing energy in an effectual manner is a challenging task in Wireless Sensor Networks. This paper presents an overview on various energy efficient routing protocols which fulfills the criteria of QoS parameters. Various energy effective and QoS based routing protocols have been compared. To improve the QoS in a network, data fusion and data accumulation is considered to be one of major energy saving technique. The routing protocols based on data aggregation, reduced cost routing and secure routing are also discussed in detail. Simulation tools like NS2, NS3, OMNET etc can be used to evaluate the network performance.
\end{abstract}

Keywords: energy efficiency, QoS, routing, Wireless Sensor Networks.

Cite this Article: Syed Mohd Ali, Dr Syeda Gauhar Fatima, Syeda Kausar Fatima and Dr Syed Abdul Sattar, Energy Efficient Routing in Wireless Sensor Networks Based on QOS, International Journal of Advanced Research in Engineering and Technology, 10(2), 2019, pp. 97-104.

http://iaeme.com/Home/issue/IJARET?Volume $=10 \&$ Issue $=2$

\section{INTRODUCTION}

\subsection{Wireless Sensor Networks}

Wireless Sensor Network (WSN) is used for monitoring and record the physical conditions of the environment and communicate the data gathered from the monitored field through wireless links. A sensor network comprises of a set of few hundreds to thousands sensor nodes which 
can perform some particular action and are battery powered. Compared to other wireless networks, the number of nodes and density of nodes comprising WSNs is huge. Due to recent technological improvements, sensor nodes have become small, inexpensive, low-power and distributed. Sensor networks have been useful in many fields like military, health, monitoring environment, industry, Science, transportation, civil infrastructure, habitat monitoring and security. While communicating the collected information, energy conservation is a key issue that has to be taken into consideration. The network is also concerned with providing the required QoS. Due to the resource constrains of wireless sensor nodes, great challenge has been placed on properties such as delay, reliability, fault tolerance and coverage. Data fusion and data accumulation should be done in order to save energy in Wireless Sensor Networks. The communication between the sensor and the base station takes place through transitional nodes which drains more energy to transmit sensed data over long distance. Many techniques have been advanced to reduce the energy consumption in these intermediate nodes. Aggregator node is one such node which can reduce the energy consumption in these transitional nodes. This process is stated as data aggregation [1-5].

\subsection{Motivation}

In WSNs, while communicating the collected information the network is concerned with providing the required QoS. QoS is the measure of service quality that the network offers to the end user or application. For efficient and consistent transmission of communication of the sensed data from the sensor field to the sink node, the network has to fulfil the required QoS. Few of the QoS parameters include data precision, accumulation delay, fault tolerance, optimum number of active sensors, coverage, energy efficiency, responsiveness, reliability, timeliness, robustness Self-Configuration Privacy and Security. QoS is also determined by calculating the throughput, delay, jitter and packet loss rate of the network. As wireless sensors carry severe energy and computational resource constrains QoS support has been challenging task in Wireless Sensor Networks [6]. An energy limitation is one of the main issue in Wireless Sensor Networks since the nodes are energized by battery power. Hence, it is essential to design an energy-aware system to ensure the durability of surveillance missions and many mechanism have been proposed recently [7-8].

\section{LITERATURE SURVEY}

Chu-Fu Wang et al., [9] proposes a network lifetime enhancement method for sink rearrangement and its study in Wireless Sensor Networks. He describes the method to conserve network lifetime while executing the sensing and reporting task. He proposed energy-aware sink relocation (EASR) methods for mobile sinks in WSNs. This method of sink rearrangement was an effective one in increasing the network lifetime by avoiding the battery energy consumption. This sink rearrangement method requires mathematical survey and effective routing protocols. Özgür B. Akan et al., [10] proposes Event-toSink Reliable Transport in Wireless Sensor Networks. He designed reliable event detection using minimum energy usage. This Event-to-Sink Reliable Transport (ESRT) protocol works by controlling the blocking of the network. Multiple event occurrences is accommodated in Wireless Sensor Networks. Here, the main function is run on sink and least functionality is shown at the source end. Hui Wang et al., [11] describes Network Lifetime Optimization in Wireless Sensor Networks. In this protocol, design of physical, medium access control and routing layers are done in such a way that their network lifetime can be increased. An iterative algorithm is also proposed for large planar networks. Here, to address the delinquent of network lifespan Time Division Multiple Admittance technique is adopted. Kareesh -Kuhn-Tucker (KKT) conditions also provide network lifetime expansion for cross layer network design. A unique algorithm has to be designed which can upsurge the network lifetime for both small and large planar networks. 
Hamid Rafiei Karkvandi et al., [12] presents Effective Lifetime-Aware Routing in Wireless Sensor Networks. This method determines the network resource stipulations such as the number of available nodes and their sensing spatial coverage. Here, the novel sensing problem is addressed and examined for various layers of the network. By using the results of various linear programming equations, normalized network lifetime can be calculated for various network environments. Jae-Wan Kim [13] describes an Intelligent Agent-based Routing Structure for Mobile Sinks in WSNs. As common moving sinks demand for frequent location inform, much of the energy consumption is due to these frequently moving and updating sink nodes. It works on data assembly and selection of efficient path techniques. This scheme shows outstanding presentation when considering a mobile sink. The proposed algorithm reduces the packet loss and signal overhead in the data communication. Distance comparison is done and new path is selected if original path is considered to consume more power. This scheme demands for fast and precise path selection technique.

Demin Wang et al., [14] describes Coverage and Lifetime Optimization of Wireless Sensor Networks using Gaussian Distribution. Coverage and lifetime are directly associated with battery power of the network. The sensors employed for this communication follow Gaussian Distribution and different dispersions. By considering various critical model coverage and lifetime can be increased in Gaussian Distribution. In Gaussian Distribution, energy saving option is not shown for sleep and wake scheduling. So, Gaussian Distribution is not an efficient one for multi hop routing protocol. Leandro et al.,[15] proposes DRINA: A Lightweight and Reliable Routing Approach for In-Network Aggregation in Wireless Sensor Networks which reduces the energy consumption of various high density nodes. Data fusion and data accumulation methods can remove the dismissal in the data and can reduce the energy ingesting and cost. Here, reduced number of messages are used for setting up a routing tree, maximized number of overlapping routes, high aggregation rate, reliable data aggregation and transmission. The proposed algorithm includes the Information Fusionbased Role Assignment (InFRA) and Shortest Path Tree (SPT) algorithms. These algorithm provide good performance in aggregation of data. Ankit Thakkar et al ., [16] elaborates on Cluster Head Election for Energy and Delay Constraint Applications of Wireless Sensor Network and proposes collection formation that results in good performance with respect to energy and interruption constraints of the network. In this cluster formation, cluster head collects and aggregates the data from member nodes and send it to the other cluster head or the base station, thus attaining good scalability. Study of the proposed algorithm was done by considering two distances Euclidean distance and multi Hop-count distance. Energy consumption was more for Euclidian distance than the multi hop count distance. The Hop count exhibited higher delay when compared to Euclidian distance.

\section{ROUTING MECHANISMS IN WIRELESS SENSOR NETWORKS}

Efficient routing protocol should control the energy consumption of the network and they should reduce the difficulty of the network. During communication between sensor nodes, these protocols should reduce the transfer delay occurring at the nodes. In this paper, we have associated various energy efficient routing protocols by upholding better QoS. If the cost is associated with the link length of the network, then the shortest path link will be the wellorganized path in communicating the data with reduced cost. The Shortest Path is the distance from any given node to the terminus node. If there is continuous data communication, bandwidth may be a limitation in shortest path route. Such shortest path routing networks should take bandwidth issue as major consideration, otherwise this network will result in highly jammed paths. This will also causes increase in delay of the network and packet losses, which in turn results in retransmission of packets and thereby increases energy consumption. So these routing protocols should also focus on all these parameters to select the shortest path such that 
it should increase the network lifespan [17]. The Dijkstra algorithm and the Bellman-Ford algorithm are the two very well known and well-defined algorithms for shortest path routing which can save power consumption. The Event-Sink Reliable Transport (ESRT) protocol scheme can attain high rate of jamming control which can also preserve energy to large extent. In this scheme, the configuration of the network is chosen very close to the optimum operating point. The main functionality is carried on sink negligible functionality at resource constrained sensor nodes. This method helps to achieve the required dependability with minimum energy consumption. Network blocking can be considerably reduced. Other methods like scheming duty cycle scheduling for sensor nodes was adopted to conserve energy. When nodes were idle without doing the sensing job, they were allowed to enter the sleep state to conserve power. This method does not effect the sensing operation of nodes[10]. Energy-Aware Sink Relocation (EASR) method adopts energy-aware routing technique for message relaying and the network lifetime of a WSN is substantially prolonged [18].

\subsection{Data Aggregation Routing Protocol in Wireless Sensor Networks}

Data aggregation is essentially done to save out from all data collected on sensors and interconnects only data that is important for the application. energy consumption and also to increase the data precision. Investigators have developed different plans to deal with the extensive data generated. The frequency of sensing and conveying the data consumes the most energy in a sensor network. The raw (i.e., sensed data) or processing (i.e., averaged sensed data) data interactions between sensor nodes in a network. Processing device data enables important data to be filtered Sensor node transmits sensed data to the base station through intermediate nodes. Since base station is too far, it drains more energy to transmit data over long distance, so the enhanced technique is to avoid direct transmission to outermost node and use only fewer nodes to communicate with the base station. These nodes are called as aggregator nodes and the process is mentioned as a data aggregation. The aggregator node collects data from multiple sensor nodes of the network, aggregates the data packet using some aggregation function and then sends the results to upper aggregator node that creates the query [19]. In many applications, WSNs encounters some security problems, so the scheme of data aggregation not only improves raw data and reduces the amount of transmission for network, but also keeps the network at a high level of security [20]. Data Routing for In-Networking Aggregation (DRINA) protocol uses some key number of messages for setting up the path and a routing tree. It consists of maximized number of overlapping routes, high aggregation rate, reliable data aggregation and transmission. This work can also be protracted to consider the spatial and sequential correlation of the aggregated data. In Privacy-preserving Data Aggregation (PDA) protocol, the privacy of both the participants and the keywords are protected. This scheme safeguards the private data, increases the throughput. The Recoverable Concealed Data Aggregation (RCDA) protocol designs the base station in such a way that, it verifies the reliability and legitimacy of all sensing data and also it performs aggregation operations on the data. So, it provides better security, increases throughput and packet delivery rate. Energy- Efficient, Secure, highly accurate and Scalable Scheme for Data Aggregation (EESSDA) scheme establishes secure channel and slicing technology to achieve secure data aggregation. This avoids encryption and decryption operations during data aggregation; instead it saves energy and gains high accuracy of aggregation results. So, it reduces time for data dispensation and it is scalable. An overhead is caused due to slicing and assembling technique since, it has to decompose data into pieces, sending data pieces to neighbor nodes and assembling their data pieces.

\subsection{QoS Based Routing Protocol in Wireless Sensor Network}

Many protocols based on refining the efficiency of various QoS parameters have been intended so far. Deterministic bounds have been given for a node-to-node delay and dependability which 
provides guarantee in terms of delay and reliability. Based on principle of network coding for retransmitting lost packets in a single hop wireless network, well-organized latency and bandwidth requirement can be attained. The loss recovery mechanism called Active Caching is used to recover the lost packets from the source to the transitional node so that intermediate information contains all the data just like source node. This retransmission improves the dependability in the network. In co-operative communication network, transmission is carried out by using a transmit node which exploits temporal and spatial diversity and reduces the packet error rates. It also reduces the number of transmissions and improves overall performance using reasoning interference management, the optimal throughput is attained by the secondary node user when primary node user accepts a retransmission-based error control scheme. Throughput is achieved by secondary node user. In the local cooperative relay for resourceful data forwarding the best local transmit node is selected and additional overhead can be effectively reduced. In one of the technique in cooperative forwarding, receiver can decode only one transmission at a time. These techniques address the impact problem. Modified CSMA/CA protocol and randomized waiting scheme is also addressed to reduce the number of retransmissions. In novel adaptive retransmission algorithm, the misclassification probability of the network can be improved. Here, the error correcting codes is designed to obtain the efficient fault-tolerant classification system. In cooperative retransmission mechanism, a node can retransmit lost packets on behalf of its neighboring node. However, lost packets can be recovered by a neighboring node, a new transmission for each transmission attempt is still required. In GORMA(Group Optimal Retransmission Medium Access Protocol), retransmission with required QoS is designed and evaluated.

\subsection{Security Based Routing in Wireless Sensor Networks}

A strong security is required in majority of sensor network applications. Major application comprises military, banking, applications working with subtle personal data which may include health, home appliances. Location and energyaware characteristics for routing is necessary to design a required protocol which gives a better transport rate, energy balancing and routing competence. Some of network layer attacks on Wireless Sensor Networks are Eavesdropping, selective forwarding, denial of service, message tampering, sinkhole attack, wormhole attack, acknowledgement fooling etc. Eavesdropping is mainly in broadcast network any opponent with a strong receiver could eavesdrop and interrupt transmitted data. Information like location of node, Message IDs, Node IDs, timestamps, application specific information can be retrieved by a burglar. Strong encryption techniques are necessary to prevent this attack. In a Denial-ofService (DoS) attack, an adversary attempts to disrupt, corrupt or destroy a network reducing the performance of the network. Message interfering is the alteration of the information to be forwarded to the destination. The Cyclic Redundancy Code (CRC) check is required at the destination. If the redundancy check fails it would result in dropping the packet. In selective forwarding attack, malicious nodes may refuse to forward certain messages and simply drop them, ensuring that they are not propagated and reduces performance. 


\section{PERFORMANCE EVALUATION OF DIFFERENT ROUTING PROTOCOLS}

TABLE 1 Comparison of Energy Efficient and QOS Based Routing Protocols

\begin{tabular}{|c|c|c|c|}
\hline Author & Algorithm/protocol & Concept & Performance \\
\hline Dong, W & $\begin{array}{c}\text { Efficient code } \\
\text { dissemination protocol }\end{array}$ & $\begin{array}{l}\text { Dynamically configures the } \\
\text { packet sizes and accurately } \\
\text { selects the sender }\end{array}$ & $\begin{array}{l}\text { Shortens the time spent in } \\
\text { selection of sender avoids } \\
\text { transmission collision and } \\
\text { transmission over poor } \\
\text { links }\end{array}$ \\
\hline Degan Zhang & $\begin{array}{l}\text { Energy balanced routing } \\
\text { method based on forward } \\
\text { aware factor }\end{array}$ & $\begin{array}{l}\text { Next hop node is selected based } \\
\text { on the link weight and forward } \\
\text { energy density }\end{array}$ & $\begin{array}{l}\text { Guarantees high QoS } \\
\text { Prolongs the function } \\
\text { lifetime }\end{array}$ \\
\hline Leandro & DRINA & $\begin{array}{l}\text { Redundant data is aggregated } \\
\text { Shortest path is established } \\
\text { between the source and the sink }\end{array}$ & $\begin{array}{l}\text { High and reliable data } \\
\text { aggregation } \\
\text { Maximum number of } \\
\text { overlapping routes. }\end{array}$ \\
\hline $\begin{array}{l}\text { Hamid Rafiei } \\
\text { Karkvandi }\end{array}$ & $\begin{array}{c}\text { Effective lifetime aware } \\
\text { routing }\end{array}$ & $\begin{array}{c}\text { Sensing spatial coverage is } \\
\text { proposed }\end{array}$ & $\begin{array}{l}\text { Improvement in QoS and } \\
\text { network lifetime. }\end{array}$ \\
\hline ui Wang & $\begin{array}{c}\text { Kareesh - } \\
\text { KuhnTucker(KKT) }\end{array}$ & $\begin{array}{c}\text { Optimal design is done on power } \\
\text { control at the physical layer the } \\
\text { design is based on cross layer } \\
\text { approach }\end{array}$ & $\begin{array}{l}\text { Optimization of network } \\
\text { lifetime. } \\
\text { Power controlling is } \\
\text { increased }\end{array}$ \\
\hline Demin Wang & $\begin{array}{l}\text { Coverage and life time } \\
\text { optimization with } \\
\text { Gaussian } \\
\text { Distribution }\end{array}$ & $\begin{array}{l}\text { Polynomial time complexity and } \\
\text { optimal deployment strategy is } \\
\text { used. Lifetime and coverage is } \\
\text { based on 2D Guassian } \\
\text { distribution }\end{array}$ & $\begin{array}{c}\text { Minimizes the network } \\
\text { cost under two constraints } \\
\square \quad \text { coverage } \\
\square \quad \text { life time. }\end{array}$ \\
\hline $\begin{array}{l}\text { OZgur B } \\
\text { Akhan }\end{array}$ & $\begin{array}{l}\text { Event to sink Reliable } \\
\text { transport in Wireless } \\
\text { Sensor Networks. }\end{array}$ & $\begin{array}{l}\text { Sink will collect the information } \\
\text { of the sensor nodes within the } \\
\text { event radius no end-to-end } \\
\text { reliability. }\end{array}$ & $\begin{array}{l}\text { Congestion control is } \\
\text { achieved. Maximization } \\
\text { of reliability and } \\
\text { conservation of energy. }\end{array}$ \\
\hline $\begin{array}{l}\text { D.Antony Arul } \\
\text { Raj }\end{array}$ & $\begin{array}{l}\text { Enhanced energy efficient } \\
\text { multipath routing }\end{array}$ & $\begin{array}{l}\text { It uses distance vector concept } \\
\text { and hop by hop routing. } \\
\text { It detects the multipath with } \\
\text { loopfree and disjoints using } \\
\text { flood-based route discovery. }\end{array}$ & $\begin{array}{c}\text { Increases lifetime of the } \\
\text { network } \\
\text { Bio inspired methods } \\
\text { have several advantages }\end{array}$ \\
\hline
\end{tabular}

In a sinkhole attack, malicious sink is created which can not only interfere the transmitted data but can also drop some important data and lead to other attacks like eavesdropping and selective forwarding. In Wormhole attack, an opponent receives messages by making a passageway and a low-latency connection in one part of the network and replays them in a different part causing some misperception in the network. In acknowledgement spoofing: An opponent can parody link layer acknowledgment for ,,,overheard ${ }^{\text {eeee }}$ packets addressed to adjacent nodes to convince the sender that a weak link is strong or that a dead or disabled node is alive. By this attack a routing protocol may select the next hop in a path using link reliability.

\section{SIMULATION}

Most of the protocols conversed above can be designed and verified using NS2 simulation tool. This simulation tool can regulate the overall performance of the designed protocols and can be 
related with other network protocols. other simulation tool includes NS3, op-net, Omnet and many others.

\section{CONCLUSIONS}

QoS in any network can be measured using the constraints such as data accuracy, aggregation delay, fault tolerance, finest number of active sensors, coverage, energy efficiency, responsiveness, reliability, throughput, timeliness, sturdiness Self-Configuration Privacy and Security. In this paper, many routing protocols in Wireless Sensor Networks are conversed based on cost, security, data aggregation and QoS requirement. Efficient routing protocol controls the energy consumption of the network and they should reduce the difficulty of the network. Different protocols based on reduced energy consumption are conferred. Many protocols can be advanced to save energy by efficiently aggregating the data. The protocols discussed in this paper can be assessed for various parameters using various simulation tools.

\section{REFERENCES}

[1] G L Wang, G H Cao, and T L Porta, "Movement-Assisted Sensor Deployment," in Proc. IEEE Inf. Commun. Conf., pp. 2469-2479, Aug. 2004.

[2] Marta and M Cardei, "Improved Sensor Network Lifetime with Multiple Mobile Sinks," J. Pervas. Mobile Comput., vol. 5, no. 5, pp. 542-555, October. 2009.

[3] P Ji, "DAST: A QoS-Aware Routing Protocol for Wireless Sensor Networks," in Proceeding of International Conferences on Embedded Software and Systems Symposia, Sichuan, pp. 259-264, July 2008.

[4] J Luo and J P Hubaux, "Joint Mobility and Routing for Lifetime Elongation in Wireless Sensor Networks," in Proc. IEEE Inf. Commun. Conf., vol. 3, pp. 1735-1746, Mar. 2005.

[5] S Basagni, I Chlamtac, V R Syrotiuk, and B A Woodward, "A Distance Routing Effect Algorithm for Mobility (DREAM),” in Proc. ACM MobiCom, pp. 76-84, Oct. 1998.

[6] B Bhuyan, H Sarma, N Sarma, A Kar and R Mall, "Quality of Service (QoS) Provisions in Wireless Sensor Networks and Related Challenges," in Journal of Wireless Sensor Network, vol. 2, no. 11, 2010.

[7] G Anastasi, M Conti, M Francesco, A Passarella, "Energy Conservation in Wireless Sensor Networks: A Survey,” in Journal of Ad Hoc Networks, vol. 7, no. 3, pp. 537-568, 2009.

[8] Degan Zhang, Guang Li, Ke Zheng, Xuechao Ming, "An Energy-Balanced Routing Method Based on Forward-Aware Factor for Wireless Sensor Networks" IEEE Transaction on Industrial Informatics, vol.10, no. 1, February 2014.

[9] Chu-Fu Wang, Jau-Der Shih, Bo-Han Pan and Tin-Yu Wu, "A Network Lifetime Enhancement Method for Sink Relocation and Its Analysis in Wireless Sensor Networks" in IEEE Sensor Journal, vol. 14, no. 6, pp. 19321934, June 2014.

[10] Özgür B Akan, and Ian F Akyildiz, IEEE “ Event-to-Sink Reliable Transport in Wireless Sensor Networks" in IEEE/ACM Transactions on Networking, vol. 13, no. 5, pp. 10331066, October 2005.

[11] Hui Wang, Nazim Agoulmine, Maode Ma, and Yanliang Jin "Network Lifetime Optimization in Wireless Sensor Networks" IEEE Journal on Selected Areas in Communication, vol. 28, no. 7, pp. 1127-1137, September 2010.

[12] Hamid Rafiei Karkvandi, Efraim Pecht, and Orly Yadid-Pecht, "Effective Lifetime-Aware Routing in Wireless Sensor Networks" IEEE Sensor Journal, vol. 11, no. 12, pp. 3359-3367, December 2011.

[13] Jae-Wan Kim, Jeong-Sik In, Kyeong Hur, Jin-Woo Kim and Doo-Seop Eom "An Intelligent Agent-based Routing Structure for Mobile Sinks in WSNs" in IEEE Transactions on Consumer Electronics, vol. 56, no. 4, pp. 2310-2316, November 2010. 
[14] Demin Wang, Bin Xie, Senior and Dharma P Agrawal, "Coverage and Lifetime Optimization of Wireless Sensor Networks with Gaussian Distribution" in IEEE Transactions on Mobile Computing, vol. 7, no. 12, pp. 1444-1458, December 2008.

[15] Leandro Aparecido Villas, Azzedine Boukerche, Heitor Soares Ramos, Horacio A.B. Fernandes de Oliveira, Regina Borges de Araujo, and Antonio Alfredo Ferreira Loureiro "DRINA: A Lightweight and Reliable Routing Approach for In-Network Aggregation" IEEE Transactions on Computers, vol. 62, no. 4, pp. 676-689, April 2013.

[16] Ankit Thakkar, Ketan Kotecha, "Cluster Head Election for Energy and Delay Constraint Applications of Wireless Sensor Network", in IEEE Transactions on Computers, vol. 62, no. 4, pp. 676-689, April 2013.

[17] Nikolaos A Pantazis, Stefanos A Nikolidakis and Dimitrios D Vergados, "Energy-Efficient Routing Protocols in Wireless Sensor Networks": A Survey Communication Surveys and Tutorials, vol. 15, no. 2, 2013.

[18] L Sun, Y Bi, and J Ma, "A Moving Strategy For Mobile Sinks In Wireless Sensor Networks," in Proc. 2nd IEEE Workshop Wireless Mesh Netw., pp. 151-153, Sep. 2006.

[19] Kiran Maraiya, Kamal Kant and Nitin Gupta, "Architecture Based Data Aggregation Technique in Wireless Sensor Networks", In International Journal on Computer Science and Engineering, vol.3, no.3, pp. 1131-1138, 2011.

[20] Taochun Wang, Xiaolin Qin and Liang Liu, "An Energy Efficient and Scalable Secure Data Aggregation for Wireless Sensor Networks", In International Journal of Distributed Sensor Networks, 2013.

[21] V. Bapuji, R. Naveen Kumar, Dr. A. Govardhan and Prof. S.S.V.N. Sarma, Maximizing Lifespan of Mobile Ad Hoc Networks with QOS Provision Routing Protocol, International Journal of Computer Engineering and Technology (IJCET), Volume 3, Issue 2, JulySeptember (2012), Volume 3, Issue 2, July- September (2012), pp. 150-156.

[22] Jayashree Agrakhed, G. S. Biradar and V. D. Mytri, Optimal QOS Routing with Prioritized Region Scheduling Over WMSN, International Journal of Computer Engineering and Technology (IJCET), Volume 3, Issue 1, January- June (2012), pp. 289-304.

[23] Bibin Mathew, Winner George and Mildred Pereira, QOS Enhancement in 4G Heterogeneous Networks Using Kalman Filter \& EWMA, International Journal of Electronics and Communication Engineering and Technology, 8(3), 2017, pp. 28-43

[24] N. Devakirubai and S. Palani, QOS Ensured Optimal Replica Placement in Graph Based Data Grids, International Journal of Computer Engineering and Technology (IJCET), Volume 4, Issue 6, November - December (2013), pp. 314-325

[25] Savitha Suresh and Lethakumary. B, Simulative Analysis of Channel and QOS Aware Scheduler to Enhance the Capacity of Multimedia LTE Networks, International Journal of Advanced Research in Engineering and Technology (IJARET), Volume 6, Issue 5, May (2015), pp. 01-08.

[26] Vijay Ukani, Tanish Zaveri and Sameer Kapadia, VAQMAC: Video Aware QOS Mac Protocol for Wireless Video Sensor Networks, International Journal of Electronics and Communication Engineering \& Technology (IJECET), Volume 5, Issue 4, April (2014), pp. $103-115$ 